OPEN ACCESS

Edited by:

Ignacio Melero,

University of Navarra, Spain

Reviewed by:

Jianxun J. Song,

Texas A\&M Health Science Center,

United States

Bin Xiong,

Huazhong University of Science and

Technology, China

${ }^{*}$ Correspondence:

Kangshun Zhu

zhksh010@163.com

${ }^{+}$These authors have contributed equally to this work and share

first authorship

Specialty section:

This article was submitted to

Cancer Immunity

and Immunotherapy,

a section of the journal

Frontiers in Immunology

Received: 04 January 2022 Accepted: 10 February 2022

Published: 01 March 2022

Citation:

Cai M, Huang W, Huang J, Shi W, Guo Y, Liang L, Zhou J, Lin L, Cao B,

Chen Y, Zhou J and Zhu K (2022)

Transarterial Chemoembolization Combined With Lenvatinib Plus PD-1 Inhibitor for Advanced Hepatocellular Carcinoma: A Retrospective Cohort Study. Front. Immunol. 13:848387. doi: 10.3389/fimmu.2022.848387

\section{Transarterial Chemoembolization Combined With Lenvatinib Plus PD-1 Inhibitor for Advanced Hepatocellular Carcinoma: A Retrospective Cohort Study}

Mingyue Cai ${ }^{1,2 t}$, Wensou Huang ${ }^{1,2 t}$, Jingjun Huang ${ }^{1,2 t}$, Wenbo Shi ${ }^{1,2}$, Yongjian Guo $^{1,2}$, Licong Liang ${ }^{1,2}$, Jingwen Zhou ${ }^{1,2}$, Liteng Lin ${ }^{1,2}$, Bihui Cao ${ }^{1,2}$, Ye Chen ${ }^{1,2}$, Juan Zhou ${ }^{3}$ and Kangshun Zhu ${ }^{1,2 *}$

${ }^{1}$ Department of Minimally Invasive Interventional Radiology, The Second Affiliated Hospital of Guangzhou Medical University,
Guangzhou, China, ${ }^{2}$ Radiology Center, The Second Affiliated Hospital of Guangzhou Medical University, Guangzhou, China,
${ }^{3}$ Department of Pharmacy, The Second Affiliated Hospital of Guangzhou Medical University, Guangzhou, China

Purpose: To investigate the efficacy and safety of transarterial chemoembolization (TACE) combined with lenvatinib plus PD-1 inhibitor (TACE-L-P) versus TACE combined with lenvatinib (TACE-L) for patients with advanced hepatocellular carcinoma (HCC).

Materials and Methods: Data of advanced HCC patients treated with TACE-L-P (TACEL-P group) or TACE-L (TACE-L group) from January 2019 to December 2020 were prospectively collected and retrospectively analyzed. The differences in overall survival (OS), progression-free survival (PFS), tumor responses (based on modified Response Evaluation Criteria in Solid Tumors) and adverse events (AEs) were compared between the two groups. Potential factors affecting OS and PFS were determined.

Results: A total of 81 patients were included in this study. Among them, 41 received TACE-L-P and 40 received TACE-L. The patients in TACE-L-P group had prolonged OS (median, 16.9 vs. 12.1 months, $P=0.009$ ), longer PFS (median, 7.3 vs. 4.0 months, $P=0.002)$ and higher objective response rate $(56.1 \% \mathrm{vs} .32 .5 \%, P=0.033)$ and disease control rate $(85.4 \%$ vs. $62.5 \%, P=0.019)$ than those in TACE-L group. Multivariate analyses revealed that the treatment option of TACE-L, main portal vein invasion and extrahepatic metastasis were the independent risk factors for OS, while TACE-L and extrahepatic metastasis were the independent risk factors for PFS. In subgroup analyses, a superior survival benefit was achieved with TACE-L-P in patients with extrahepatic metastasis or tumor number $>3$ but not in those with main portal vein invasion. 
The incidence and severity of AEs in TACE-L-P group were comparable to those in TACE-L group (any grade, $92.7 \%$ vs. 95.0\%, $P=1.000$; grade 3, 36.6\% vs. 32.5\%, $P=0.699$ ).

Conclusion: TACE-L-P significantly improved survival over TACE-L with an acceptable safety profile in advanced HCC patients, especially those with extrahepatic metastasis or tumor number $>3$ but without main portal vein invasion.

Keywords: hepatocellular carcinoma, transarterial chemoembolization, lenvatinib, immune checkpoint inhibitor, PD-1 inhibitor, combined therapy

\section{INTRODUCTION}

Hepatocellular carcinoma (HCC), representing 75\%-85\% of primary liver cancer, is one of the most prevalent and fatal malignancies worldwide (1). Although surgical resection, ablation and liver transplantation may provide curative potential for HCC, a majority of patients are diagnosed with advanced disease which is not amenable for these approaches and thus bear a poor prognosis with an expected median survival of 6-8 months (2-4).

The multikinase inhibitors sorafenib and lenvatinib are recommended as the first-line treatment of advanced HCC (24 ) on the basis of randomized trials demonstrating longer survival with sorafenib versus placebo $(5,6)$ and noninferiority of lenvatinib to sorafenib (7). However, the efficacy of monotherapy with these drugs is modest, and only a small survival benefit of about 3 months can be achieved with oral sorafenib (8). In this setting, transarterial chemoembolization (TACE) has been applied to providing local disease control in patients with acceptable liver function and tumor burden. It is supposed that the antiangiogenic agents in combination with TACE may effectively offset the post-TACE hypoxia-induced angiogenesis and, therefore, provide a superior antitumor effect for $\operatorname{HCC}(9,10)$. In fact, many studies have suggested improved outcomes of this combination treatment compared with the use of a single drug or TACE alone for advanced HCC (11-14). But unfortunately, there still remained limited treatment responses with unsatisfied survival prolongation (11-13).

Recently, immune checkpoint inhibitors, including programmed death 1 (PD-1) and programmed death ligand 1 (PD-L1) inhibitors, have exhibited a promising clinical benefit to advanced HCC patients (15). Although phase III trials for antiPD-1 monotherapy failed to meet their primary survival endpoints $(16,17)$, the studies testing combined treatments with PD-1/PDL1 inhibitor and antiangiogenic agent showed exciting results (1820). In a recent phase Ib study for evaluating the combination of lenvatinib and pembrolizumab (an anti-PD-1 antibody) in firstline treatment of unresectable HCC, an objective response rate (ORR) of $46.0 \%$ per modified Response Evaluation Criteria in Solid Tumors (mRECIST) and a median overall survival (OS) of 22.0 months were achieved (20). These impressive results suggested a promising therapeutic potential of the combination of lenvatinib plus PD-1 inhibitor in patients with HCC.

Since TACE possesses a local anticancer effect and may facilitate antitumor immunity but inevitably induces post-TACE angiogenesis $(10,21)$, and lenvatinib has an immunomodulatory effect on tumor microenvironment besides antiangiogenesis (22,
23), combining TACE and lenvatinib plus PD-1 inhibitor (TACEL-P) may contribute to a synergistic anticancer activity for HCC. Accordingly, we hypothesized that the comprehensive therapy of TACE-L-P would be an effective treatment strategy for advanced HCC. Thus, we conducted this retrospective study to evaluate the efficacy and safety of TACE-L-P versus TACE combined with lenvatinib (TACE-L) in the patients with advanced HCC.

\section{MATERIALS AND METHODS}

\section{Study Design and Patient Selection}

This study was approved by our institutional review board, and written informed consent was obtained from every patient. Data of consecutive patients with advanced HCC who received TACE-L-P or TACE-L at our institution between January 2019 and December 2020 were prospectively collected and retrospectively analyzed.

The inclusion criteria for this study were as follows: 1) age between 18 and 75 years; 2) confirmed diagnosis with $\operatorname{HCC~}(2,4$, 24) accompanied by macrovascular invasion and/or extrahepatic metastasis (BCLC stage C or CNLC Stage IIIa/IIIb); 3) tumor recurrence after curative resection or ablation was allowed; 4) Eastern Cooperative Oncology Group performance status (ECOG PS) of $\leq 1$; and 5) Child-Pugh class A/B. Patients were excluded if they 1 ) had central nervous system metastasis; 2) had history of organ transplantation; 3) previously received TACE, hepatic arterial infusion chemotherapy (HAIC), radiotherapy or systemic therapy; 4) had other malignancies in addition to HCC; or 5) had severe medical comorbidities including severe cardiac, pulmonary, renal or coagulation dysfunction.

All laboratory test data were collected within 3 days before the initial treatment. Contrast-enhanced computed tomography (CT) or magnetic resonance imaging (MRI) was performed within 7 days before the initial treatment.

\section{TACE Procedure}

The patients received either conventional TACE (cTACE) or drug-eluting bead TACE (DEB-TACE) according to their own choice. For CTACE, an emulsion of 5-20 mL Lipiodol (Guerbet, Paris, France) mixed with 20-60 mg pirarubicin (Hisun Pfizer Pharmaceuticals, Fuyang, China) was administered into the tumor-feeding vessels, followed by embolization with polyvinyl alcohol particles (90-500 $\mu \mathrm{m}$; Cook, Bloomington, Indiana, USA). For DEB-TACE, CalliSpheres (Hengrui Medical, Suzhou, China) or DC Bead (Biocompatibles, Farnham, Surrey, $\mathrm{UK}$ ) with 100-300 $\mu \mathrm{m}$ in diameter was used as the drug carrier 
and embolization agent. Typically, one vial of the beads was loaded with $60 \mathrm{mg}$ pirarubicin. If blushed tumors were still visible after the embolization with one vial of beads, regular microspheres (8spheres, Hengrui Medical, Suzhou, China; Embosphere, Biosphere Medical, Roissy en France, France) with diameters of 100-700 $\mu \mathrm{m}$ were additionally injected (25).

During TACE, superselective catheterization was performed, and the embolization end point was blood stasis of the tumorfeeding arteries. In patients with huge or bilobar multiple lesions, in order to reduce the risk of complications, the embolization end point was not achieved in the initial TACE but in the second or third TACE session (26). In the case of arterioportal or arteriovenous fistula, the fistula would be embolized with 300$710 \mu \mathrm{m}$ polyvinyl alcohol particles before administration of the drug-oil emulsion or drug-loaded beads.

TACE was repeated "on demand" upon the demonstration of viable tumor by follow-up CT or MRI in patients without deteriorated performance status or organ function.

\section{Lenvatinib and PD-1 Inhibitor Administration}

Lenvatinib (Eisai, Tokyo, Japan) and PD-1 inhibitor was initiated within 7 days after the first TACE. Lenvatinib at a dose of $12 \mathrm{mg}$ (bodyweight $\geq 60 \mathrm{~kg}$ ) or $8 \mathrm{mg}$ (bodyweight $<60 \mathrm{~kg}$ ) was orally administered once a day. The PD-1 inhibitor sintilimab (Innovent Biologics, Suzhou, China), tislelizumab (BeiGene,
Shanghai, China) or camrelizumab (Hengrui Pharma, Lianyungang, China) was injected intravenously at $200 \mathrm{mg}$ once every 3 weeks. The interruption and discontinuation of drug administration depended on the presence and severity of toxicities according to the drug directions.

\section{Follow-Up}

Regular follow-up was conducted for all patients at a 3-6-week interval after the initial treatment. Each follow-up session included a detail history, physical examination, hematologic and biochemical tests, contrast-enhanced abdominal CT or MRI, chest CT, and other imaging examination if clinically indicated. The final follow-up ended on June 30, 2021.

During follow-up, the treatment of TACE-L-P or TACE-L was discontinued in cases of intolerable toxicity, progressive disease (PD) or change of treatment plan. And, the choice of the subsequent treatment, such as second-line targeted agent, PD-1 inhibitor (for the patients treated with TACE-L), radiotherapy (including iodine-125 seed brachytherapy), HAIC or best supportive care, was determined according to the results of discussion by our multidisciplinary team and the patients' request.

\section{Assessments and Outcomes}

OS and progression-free survival (PFS) were compared between TACE-L-P group and TACE-L group. OS was defined as the time from treatment initiation until death by any reason. PFS was

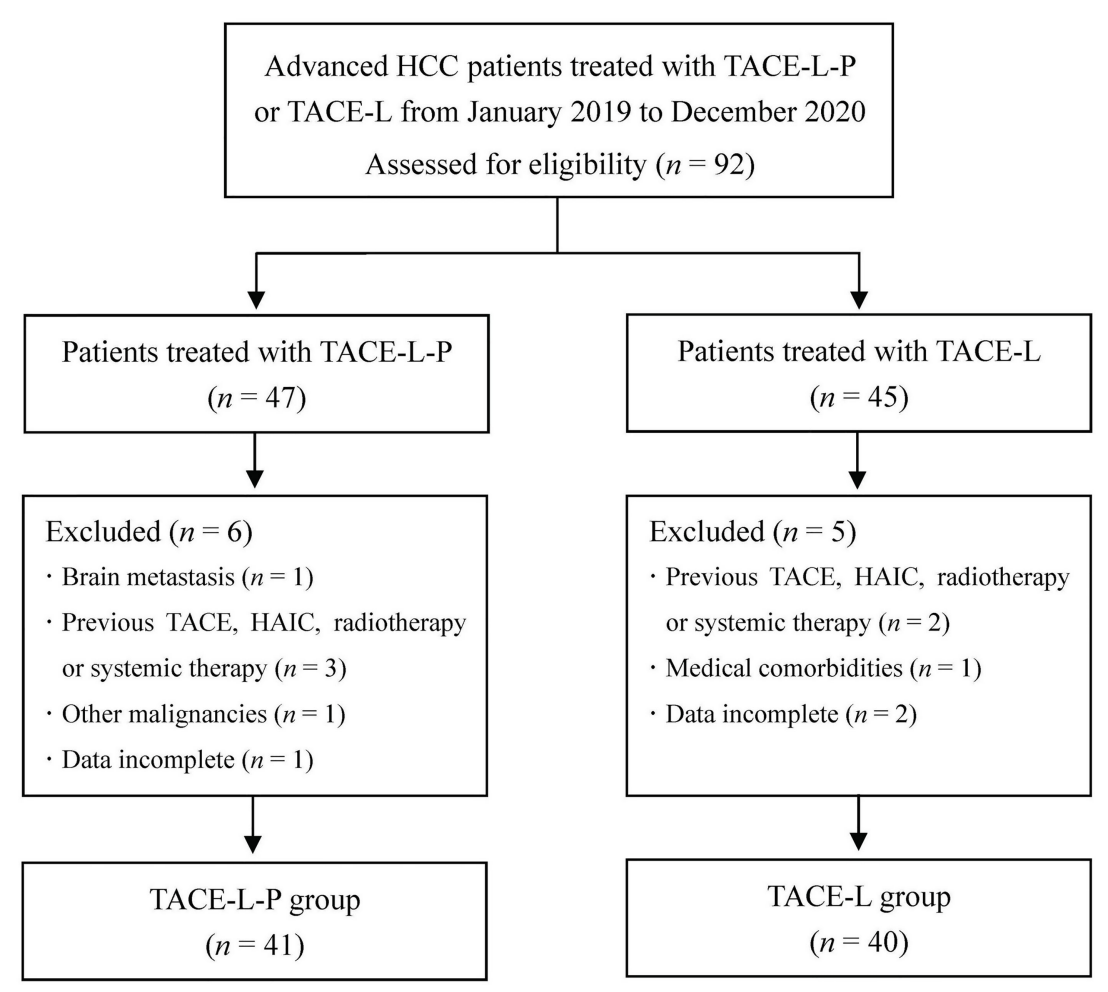

FIGURE 1 | Flow diagram of patient enrollment. HCC, hepatocellular carcinoma; TACE-L-P, transarterial chemoembolization combined with lenvatinib plus PD-1 inhibitor; TACE-L, transarterial chemoembolization combined with lenvatinib; TACE, transarterial chemoembolization; HAIC, hepatic arterial infusion chemotherapy. 
defined as the time interval from treatment initiation to the first occurrence of PD or death, whichever occurred first.

Tumor responses were categorized as complete response (CR), partial response $(\mathrm{PR})$, stable disease $(\mathrm{SD})$ or $\mathrm{PD}$ according to mRECIST. Overall tumor response referred to the assessment of changes in tumor burden inside and outside the liver, while intrahepatic tumor response only included the assessment of changes in tumor burden inside the liver. ORR was defined as the percentage of patients who had a best tumor response rating of CR and PR. Disease control rate (DCR) was defined as the percentage of patients who had a best tumor response rating of CR, PR and SD.

Adverse events (AEs) related to treatments were recorded and assessed based on Common Terminology Criteria for Adverse Events (CTCAE) version 5.0. Postembolization syndrome (manifested by fever, abdominal pain, nausea, vomiting and increased white blood cell count) and transient abnormalities of liver enzyme after TACE $(27,28)$ were expected and would resolve within a short time, and therefore, they were not documented separately.

\section{Statistical Analyses}

Categorical data were expressed as number of patients (percentage). Quantitative data were expressed as mean \pm standard deviation and median (range) for normally and nonnormally distributed variables, respectively. Categorical data between the two groups were compared using $\chi^{2}$ test or Fisher's exact test, as appropriate. Quantitative data were compared using Student's $t$-test or Mann-Whitney U test, as appropriate. Survival curves were analyzed by Kaplan-Meier method using

TABLE 1 | Baseline characteristics of the patients.

\begin{tabular}{|c|c|c|c|}
\hline Characteristic & TACE-L-P group $(n=41)$ & TACE-L group $(n=40)$ & $P$ \\
\hline Sex & & & 0.309 \\
\hline Female & $4(9.8)$ & $7(17.5)$ & \\
\hline Male & 37 (90.2) & 33 (82.5) & \\
\hline Age (years) & $51.9 \pm 10.3$ & $54.6 \pm 11.0$ & 0.263 \\
\hline ECOG PS & & & 0.274 \\
\hline 1 & $8(19.5)$ & $12(30.0)$ & \\
\hline 0 & $33(80.5)$ & $28(70.0)$ & \\
\hline HBsAg & & & 0.779 \\
\hline Positive & $35(85.4)$ & $35(87.5)$ & \\
\hline Negative & $6(14.6)$ & $5(12.5)$ & \\
\hline Child-Pugh class & & & 0.309 \\
\hline B & $4(9.8)$ & $7(17.5)$ & \\
\hline A & 37 (90.2) & $33(82.5)$ & \\
\hline AFP level ( $\mu \mathrm{g} / \mathrm{L})$ & & & 0.733 \\
\hline$\geq 400$ & $21(51.2)$ & $22(55.0)$ & \\
\hline$<400$ & $20(48.8)$ & $18(45.0)$ & \\
\hline PIVKA-II (mAU/ml) & & & 0.517 \\
\hline$\geq 400$ & 27 (65.9) & $29(72.5)$ & \\
\hline$<400$ & $14(34.1)$ & $11(27.5)$ & \\
\hline Recurrent tumor & & & 0.362 \\
\hline No & $35(85.4)$ & $31(77.5)$ & \\
\hline Yes & $6(14.6)$ & $9(22.5)$ & \\
\hline Number of tumors & & & 0.439 \\
\hline$>3$ & $23(56.1)$ & 19 (47.5) & \\
\hline$\leq 3$ & $18(43.9)$ & $21(52.5)$ & \\
\hline Tumor distribution & & & 0.939 \\
\hline Bilobar & $28(68.3)$ & $27(67.5)$ & \\
\hline Unilobar & $13(31.7)$ & $13(32.5)$ & \\
\hline Largest tumor size (cm) & $12.3 \pm 4.8$ & $13.6 \pm 5.1$ & 0.218 \\
\hline Main portal vein invasion & & & 0.441 \\
\hline Yes & 15 (36.6) & $18(45.0)$ & \\
\hline No & $26(63.4)$ & $22(55.0)$ & \\
\hline Hepatic vein invasion & & & 0.581 \\
\hline Yes & $12(29.3)$ & $14(35.0)$ & \\
\hline No & $29(70.7)$ & $26(65.0)$ & \\
\hline Extrahepatic metastasis & & & 0.585 \\
\hline Yes & $17(41.5)$ & $19(47.5)$ & \\
\hline No & $24(58.5)$ & $21(52.5)$ & \\
\hline TACE technique & & & 0.223 \\
\hline cTACE & $17(41.5)$ & $22(55.0)$ & \\
\hline DEB-TACE & $24(58.5)$ & $18(45.0)$ & \\
\hline
\end{tabular}

Data were presented as $n$ (\%) or mean \pm standard deviation. TACE-L-P, transarterial chemoembolization combined with lenvatinib plus PD-1 inhibitor; TACE-L, transarterial chemoembolization combined with lenvatinib; ECOG PS, Eastern Cooperative Oncology Group Performance Status; HBsAg, hepatitis B surface antigen; AFP, $\alpha$-fetoprotein; PIVKA-II, protein induced by vitamin K absence or antagonist-Il; TACE, transarterial chemoembolization; CTACE, conventional transarterial chemoembolization; DEB-TACE, drug-eluting bead transarterial chemoembolization. 
log-rank test. Variables with $P<0.10$ in univariate analysis were entered into a multivariate analysis using Cox regression model to identify the independent prognostic factors for OS and PFS. All statistical analyses were performed with SPSS Statistics version 22 (IBM, Armonk, New York, USA). All statistical tests were twotailed, $P<0.05$ was considered statistically significant.

\section{RESULTS}

\section{Study Population}

During the study period, 92 patients with advanced HCC who received TACE-L-P or TACE-L were screened for eligibility. Of these patients, 11 were excluded because they met the excluded criteria (Figure 1). Finally, 81 patients were included in this study (41 in the TACE-L-P group and 40 in the TACE-L group). Detailed baseline characteristics of the patients were summarized in Table 1. In both groups, about half of the patients had more than 3 intrahepatic tumors at diagnosis. The mean largest tumor size of TACE-L-P group and TACE-L group was $12.3 \pm 4.8 \mathrm{~cm}$ and $13.6 \pm 5.1 \mathrm{~cm}$, respectively. Two groups were comparable in the demographic, clinical and tumor characteristics.

The patients in TACE-L-P group underwent a total of 134 TACE procedures, with a median of 3 (range, 1-7). While the patients in TACE-L group underwent a total of 95 TACE procedures, with a median of 2 (range, 1-6). The mean duration of lenvatinib administration was $7.4 \pm 3.8$ (range, 1.215.6) months in TACE-L-P group and $4.3 \pm 3.0$ (range, 0.9-11.9) months in the TACE-L group $(P<0.001)$. In the TACE-L-P group, the cycles of $\mathrm{PD}-1$ inhibitor injection ranged from 2 to 22 , with a mean of 9.9. The categories of PD-1 inhibitor the patients received were as follows: sintilimab for 30 (73.2\%), tislelizumab for 6 (14.6\%) and camrelizumab for 5 (12.2\%).

\section{Survival}

The follow-up duration ranged from 4.6 to 29.8 months, with a median of 13.7 months. During follow-up, 27 patients (65.9\%) in the TACE-L-P group and 30 patients $(75.0 \%)$ in the TACE-L group died. Compared with the patients in TACE-L group, the patients in TACE-L-P group had significantly better survival outcomes (Figure 2). The median OS was 16.9 (95\% confidence interval [CI 14.9-18.8) months in TACE-L-P group and 12.1 (95\% CI 10.7-13.5) months in TACE-L group $(P=0.009)$. The median PFS was 7.3 (95\% CI 6.0-8.7) months in TACE-L-P group and 4.0 (95\% CI 2.7-5.3) months in TACE-L group $(P=0.002)$. Additionally, the median OS and PFS of the patients treated with sintilimab were comparable to those of the patients treated with tislelizumab/camrelizumab (OS, 17.0 months [95\% CI 14.4-19.6] vs. 16.9 months [95\% CI 9.6-24.1], $P=0.210$; PFS, 7.5 months [ $95 \%$ CI 6.8-8.2] vs. 6.2 months [95\% CI 4.6-7.7], $P=0.381$ ) in the TACE-L-P group (Supplementary Figure 1).

\section{Prognostic Factors Analysis}

Based on the results of the univariate and multivariate analyses (Table 2), treatment option (TACE-L vs. TACE-L-P; hazard ratio $[\mathrm{HR}]=2.065,95 \% \mathrm{CI} 1.208-3.533, P=0.008)$, extrahepatic metastasis (present vs. absent; HR=2.041, 95\% CI 1.183-3.520, $P=0.010$ ) and main portal vein invasion (yes vs. no; $\mathrm{HR}=1.867$, 95\% CI 1.089-3.200, $P=0.023$ ) were identified as the independent prognostic factors for OS. In addition, treatment option $(\mathrm{HR}=2.243$, 95\% CI 1.344-3.743, $P=0.002)$ and extrahepatic metastasis $(\mathrm{HR}=2.244,95 \% \mathrm{CI} 1.365-3.689, P=0.001)$ were also identified as the independent prognostic factors for PFS.

Subgroup analyses of factors for OS indicated that TACE-L-P treatment could provide a superior survival benefit in patients with no main portal vein invasion, tumor number $>3$ or extrahepatic metastasis, but failed to have a clinical benefit in
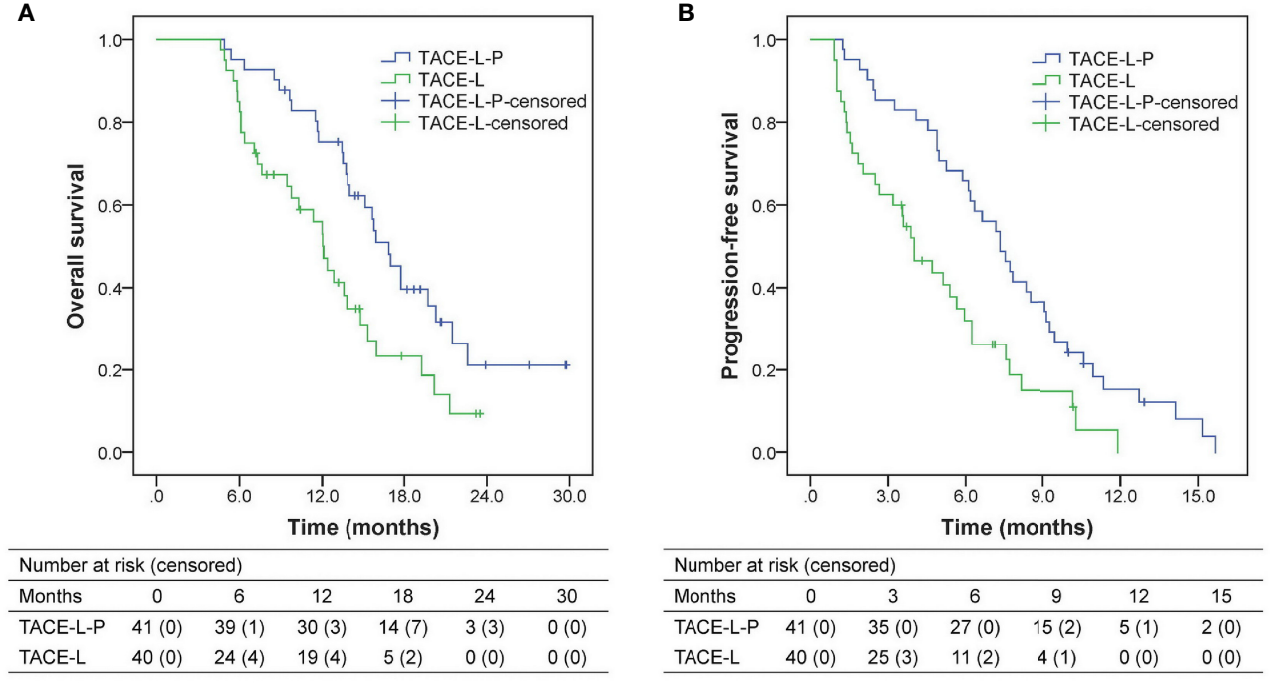

FIGURE 2 | Kaplan-Meier analyses of overall survival (A) and progression-free survival (B) according to treatment groups. TACE-L-P, transarterial chemoembolization combined with lenvatinib plus PD-1 inhibitor; TACE-L, transarterial chemoembolization combined with lenvatinib. 
TABLE 2 | Analyses of prognostic factors for survival.

\begin{tabular}{|c|c|c|c|c|c|c|c|c|}
\hline \multirow[t]{2}{*}{ Factor } & \multicolumn{4}{|c|}{ Overall survival } & \multicolumn{4}{|c|}{ Progression-free survival } \\
\hline & HR (95\% Cl) & $P$ & HR (95\% Cl) & $P$ & HR (95\% Cl) & $P$ & HR (95\% Cl) & $P$ \\
\hline \multicolumn{9}{|l|}{ Sex } \\
\hline Female/Male & 1.459 (0.713-2.984) & 0.301 & & & $0.951(0.450-2.009)$ & 0.895 & & \\
\hline \multicolumn{9}{|l|}{ Age (years) } \\
\hline $1 / 0$ & $1.587(0.897-2.809)$ & 0.113 & & & $1.271(0.748-2.162)$ & 0.376 & & \\
\hline \multicolumn{9}{|l|}{ HBsAg } \\
\hline Positive/Negative & $1.632(0.699-3.810)$ & 0.257 & & & $1.045(0.533-2.046)$ & 0.899 & & \\
\hline \multicolumn{9}{|l|}{ Child-Pugh class } \\
\hline $\mathrm{B} / \mathrm{A}$ & $1.698(0.800-3.606)$ & 0.168 & & & $1.243(0.631-2.448)$ & 0.530 & & \\
\hline \multicolumn{9}{|l|}{ AFP level ( $\mu \mathrm{g} / L)$} \\
\hline \multicolumn{9}{|l|}{ Number of tumors } \\
\hline$>3 / \leq 3$ & $1.528(0.893-2.615)$ & 0.122 & & & $1.177(0.733-1.888)$ & 0.500 & & \\
\hline \multicolumn{9}{|l|}{ Tumor distribution } \\
\hline Bilobar/Unilobar & $1.144(0.647-2.022)$ & 0.643 & & & $1.033(0.623-1.711)$ & 0.900 & & \\
\hline \multicolumn{9}{|l|}{ Largest tumor size (cm) } \\
\hline$\geq 10 /<10$ & $1.531(0.857-2.733)$ & 0.150 & & & $1.388(0.836-2.304)$ & 0.205 & & \\
\hline \multicolumn{9}{|l|}{ Main portal vein invasion } \\
\hline Yes/No & $1.638(0.970-2.767)$ & 0.065 & $1.867(1.089-3.200)$ & 0.023 & $1.025(0.635-1.653)$ & 0.920 & & \\
\hline \multicolumn{9}{|l|}{ Hepatic vein invasion } \\
\hline Yes/No & $1.263(0.721-2.211)$ & 0.414 & & & $1.315(0.783-2.206)$ & 0.300 & & \\
\hline \multicolumn{9}{|l|}{ Extrahepatic metastasis } \\
\hline Yes/No & $1.710(1.013-2.888)$ & 0.045 & $2.041(1.183-3.520)$ & 0.010 & 2.125 (1.313-3.438) & 0.002 & 2.337 (1.430-3.820) & 0.001 \\
\hline
\end{tabular}

Analyses were performed using Cox proportional hazard regression model. HR, hazard ratio; Cl, confidence interval; ECOG PS, Eastern Cooperative Oncology Group Performance Status; HBsAg, hepatitis B surface antigen; AFP, $\alpha$-fetoprotein; PIVKA-II, protein induced by vitamin $K$ absence or antagonist-II; TACE-L, transarterial chemoembolization combined with

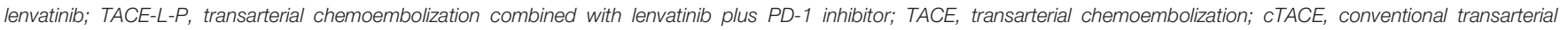
chemoembolization; DEB-TACE, drug-eluting bead transarterial chemoembolization.

patients with main portal vein invasion, tumor number $\leq 3$ or no extrahepatic metastasis (Figure 3).

\section{Tumor Responses}

Tumor responses for patients in the two groups were shown in Figure 4. The ORR of both overall tumor $(56.1 \%$ vs. $32.5 \%$, $P=0.033)$ and intrahepatic tumor $(65.9 \%$ vs. $37.5 \%, P=0.011)$ was higher in the TACE-L-P group than in the TACE-L group. A higher DCR was also achieved in TACE-L-P group when compared with TACE-L group (overall tumor, $85.4 \%$ vs. $62.5 \%$, $P=0.019$; intrahepatic tumor, $95.1 \%$ vs. $77.5 \%, P=0.021)$. In addition, the ORR (overall tumor, $56.7 \%$ vs. $54.5 \%, P=1.000$; intrahepatic tumor, $66.7 \%$ vs. $63.6 \%, P=1.000$ ) and DCR (overall tumor, $86.7 \%$ vs. $81.8 \%, P=1.000$; intrahepatic tumor, $96.7 \%$ vs. $90.9 \%, P=0.470$ ) of the patients treated with sintilimab were similar to those of the patients treated with tislelizumab/ camrelizumab in the TACE-L-P group (Supplementary Table 1).

\section{Safety}

In total, treatment-related AEs were observed in 76 of the 81 patients (93.8\%), and no grade 4/5 AEs occurred (Table 3). The frequency and severity of AEs was similar between the TACE-L$\mathrm{P}$ group and the TACE-L group (any grade, $92.7 \%$ vs. $95.0 \%$, $P=1.000$; grade $3,36.6 \%$ vs. $32.5 \%, P=0.699$ ). The AEs related to TACE, including ascites, pleural effusion, inguinal hematoma and intrahepatic biliary dilatation/biloma, were mild ( $\leq$ grade 2 ) and occurred in $6(14.6 \%)$ and $7(17.5 \%)$ patients in the TACE-L-P group and the TACE-L group $(P=0.725)$, respectively. The AEs related to lenvatinib and/or $\mathrm{PD}-1$ inhibitor occurred in $38(92.7 \%)$ and $37(90.0 \%)$ patients in the TACE-L-P group and the TACE-L group $(P=1.000)$, respectively. In the TACE-L-P group, the incidences of overall and grade 3 AEs in the patients treated with sintilimab were similar to those in the patients treated with tislelizumab/camrelizumab (any grade, $93.3 \%$ vs. $90.9 \%, P=1.000$; grade 3, $36.7 \%$ vs. $36.4 \%, P=1.000)$. 


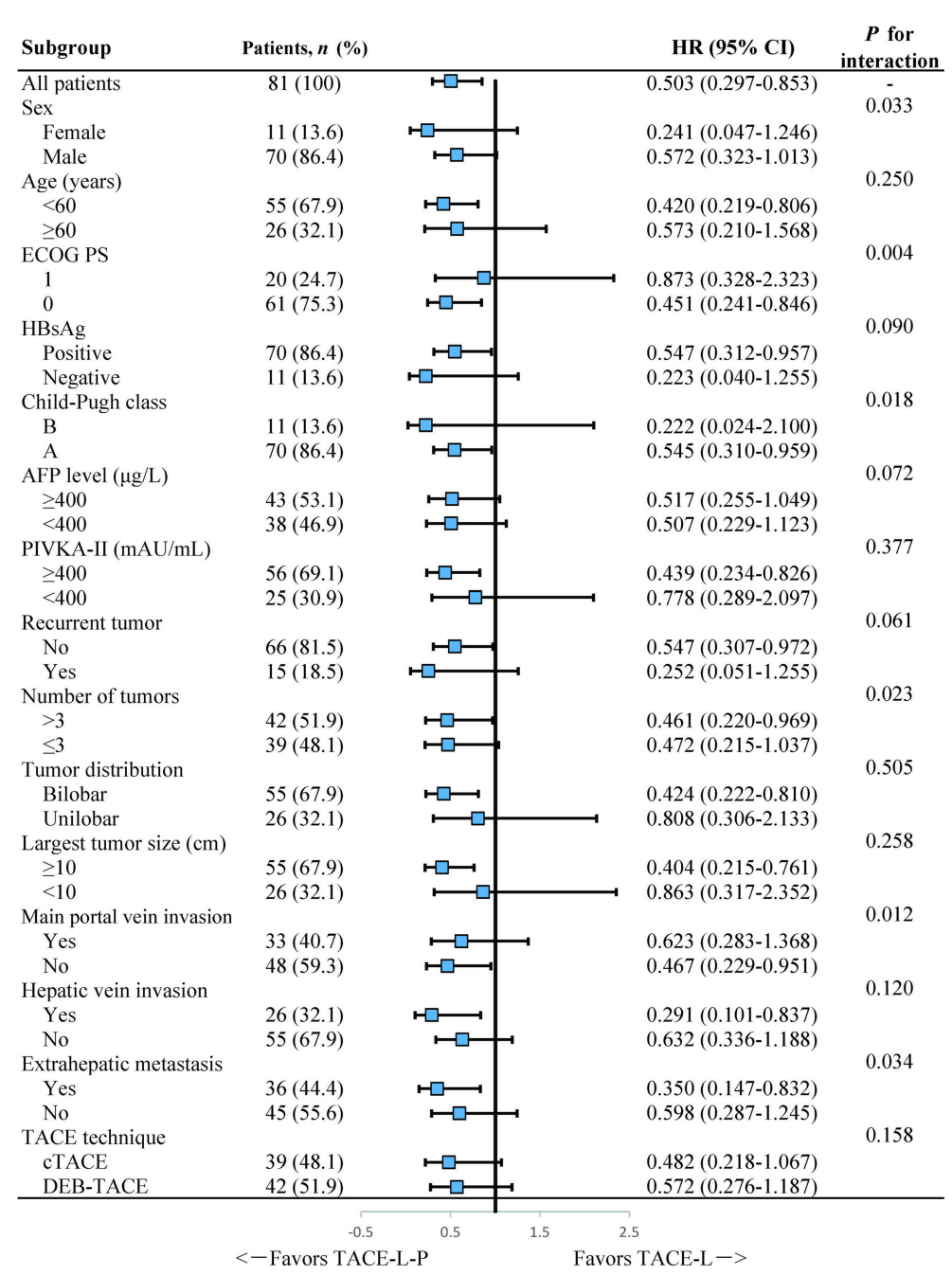

FIGURE 3 | Forest plot of the subgroup analyses for overall survival. HR, hazard ratio; Cl, confidence interval; ECOG PS, Eastern Cooperative Oncology Group Performance Status; HBsAg, hepatitis B surface antigen; AFP, $\alpha$-fetoprotein; PIVKA-II, protein induced by vitamin K absence or antagonist-II; TACE, transarterial chemoembolization; CTACE, conventional transarterial chemoembolization; DEB-TACE, drug-eluting bead transarterial chemoembolization; TACE-L-P, transarterial chemoembolization combined with lenvatinib plus PD-1 inhibitor; TACE-L, transarterial chemoembolization combined with lenvatinib.

A

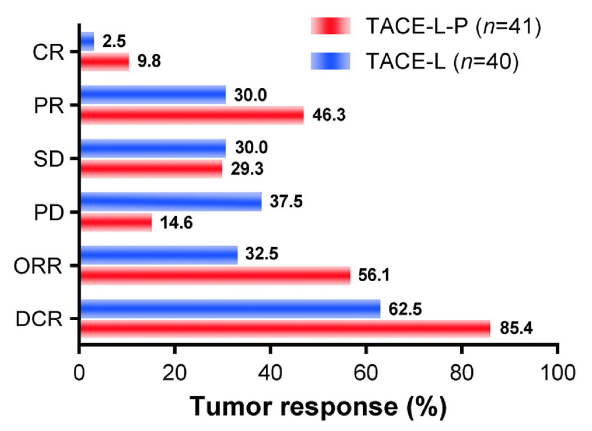

B

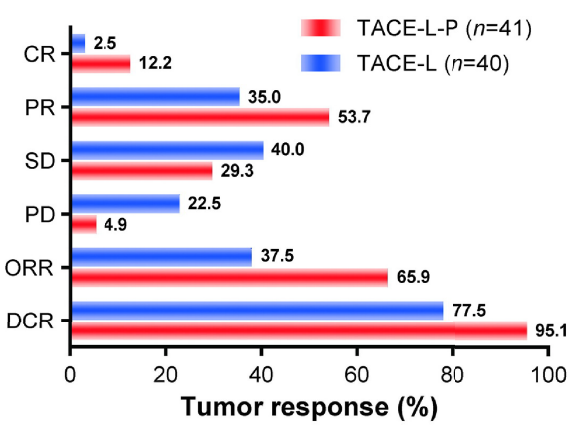

FIGURE 4 | Treatment responses of overall tumor (A) and intrahepatic tumor (B) for the two groups. TACE-L-P, transarterial chemoembolization combined with lenvatinib plus PD-1 inhibitor; TACE-L, transarterial chemoembolization combined with lenvatinib; CR, complete response; PR, partial response; SD, stable disease; PD, progressive disease; ORR, objective response rate; DCR, disease control rate. 
TABLE 3 | Treatment-related adverse events in the two groups.

\begin{tabular}{|c|c|c|c|c|c|c|}
\hline \multirow[t]{2}{*}{ Adverse events } & \multicolumn{3}{|c|}{ Any grade } & \multicolumn{3}{|c|}{ Grade 3} \\
\hline & TACE-L-P group ( $n=41)$ & TACE-L group $(n=40)$ & $\boldsymbol{P}$ & TACE-L-P group $(n=41)$ & TACE-L group $(n=40)$ & $\boldsymbol{P}$ \\
\hline Total & $38(92.7)$ & $38(95.0)$ & 1.000 & $15(36.6)$ & $13(32.5)$ & 0.699 \\
\hline Related to TACE & $6(14.6)$ & $7(17.5)$ & 0.725 & $0(0.0)$ & $0(0.0)$ & - \\
\hline New ascites & $3(7.3)$ & $2(5.0)$ & 1.000 & $0(0.0)$ & $0(0.0)$ & - \\
\hline Pleural effusion & $2(4.9)$ & $2(5.0)$ & 1.000 & $0(0.0)$ & $0(0.0)$ & - \\
\hline Inguinal hematoma & $2(4.9)$ & $3(7.5)$ & 0.977 & $0(0.0)$ & $0(0.0)$ & - \\
\hline Biliary injury ${ }^{\dagger}$ & $1(2.4)$ & $2(5.0)$ & 0.983 & $0(0.0)$ & $0(0.0)$ & - \\
\hline Related to drug ${ }^{\star}$ & $38(92.7)$ & $37(92.5)$ & 1.000 & 15 (36.6) & $13(32.5)$ & 0.699 \\
\hline Hypertension & $16(39.0)$ & $14(35.0)$ & 0.708 & $9(22.0)$ & $8(20.0)$ & 0.829 \\
\hline Weight loss & $14(34.1)$ & $11(27.5)$ & 0.517 & $1(2.4)$ & $0(0.0)$ & 1.000 \\
\hline Diarrhea & $13(31.7)$ & $13(32.5)$ & 0.939 & $0(0.0)$ & $0(0.0)$ & - \\
\hline Hand-foot syndrome & $11(26.8)$ & $13(32.5)$ & 0.576 & $0(0.0)$ & $0(0.0)$ & - \\
\hline Fatigue & $11(26.8)$ & $9(22.5)$ & 0.651 & $1(2.4)$ & $0(0.0)$ & 1.000 \\
\hline Elevated AST & $11(26.8)$ & $8(20.0)$ & 0.468 & $0(0.0)$ & $1(2.5)$ & 0.494 \\
\hline Decreased appetite & $10(24.4)$ & $9(22.5)$ & 0.841 & $1(2.4)$ & $1(2.5)$ & 1.000 \\
\hline Hypothyroidism & $10(24.4)$ & $8(20.0)$ & 0.635 & $0(0.0)$ & $0(0.0)$ & - \\
\hline Elevated ALP & $9(22.0)$ & $11(27.5)$ & 0.563 & $0(0.0)$ & $0(0.0)$ & - \\
\hline Hypoalbuminemia & $9(22.0)$ & $8(20.0)$ & 0.829 & $0(0.0)$ & $0(0.0)$ & - \\
\hline Abdominal pain & $9(22.0)$ & $8(20.0)$ & 0.829 & $0(0.0)$ & $1(2.5)$ & 0.494 \\
\hline Pruritus & $9(22.0)$ & $4(10.0)$ & 0.143 & $0(0.0)$ & $0(0.0)$ & - \\
\hline Elevated ALT & 8 (19.5) & $9(22.5)$ & 0.741 & $1(2.4)$ & $0(0.0)$ & 1.000 \\
\hline Thrombocytopenia & 8 (19.5) & $8(20.0)$ & 0.956 & $1(2.4)$ & $2(5.0)$ & 0.983 \\
\hline Neutropenia & 8 (19.5) & $6(15.0)$ & 0.591 & $2(4.9)$ & $2(5.0)$ & 1.000 \\
\hline Proteinuria & 8 (19.5) & $6(15.0)$ & 0.591 & $0(0.0)$ & $0(0.0)$ & - \\
\hline Rash & 8 (19.5) & $4(10.0)$ & 0.228 & $0(0.0)$ & $0(0.0)$ & - \\
\hline Anemia & $7(17.1)$ & $5(12.5)$ & 0.562 & $0(0.0)$ & $1(2.5)$ & 0.494 \\
\hline Lymphopenia & $7(17.1)$ & $4(10.0)$ & 0.353 & $1(2.4)$ & $0(0.0)$ & 1.000 \\
\hline Elevated TBi & $6(14.6)$ & $7(17.5)$ & 0.725 & $1(2.4)$ & $1(2.5)$ & 1.000 \\
\hline Nausea & $6(14.6)$ & $6(15.0)$ & 0.963 & $0(0.0)$ & $0(0.0)$ & - \\
\hline Elevated GGT & $6(14.6)$ & $5(12.5)$ & 0.779 & $0(0.0)$ & $0(0.0)$ & - \\
\hline Ventosity & $6(14.6)$ & $5(12.5)$ & 0.779 & $1(2.4)$ & $0(0.0)$ & 1.000 \\
\hline Arthralgia & $6(14.6)$ & $5(12.5)$ & 0.779 & $0(0.0)$ & $0(0.0)$ & - \\
\hline Vomiting & 5 (12.2) & $6(15.0)$ & 0.713 & $0(0.0)$ & $0(0.0)$ & - \\
\hline Gingival bleeding & 5 (12.2) & $6(15.0)$ & 0.713 & $0(0.0)$ & $0(0.0)$ & - \\
\hline Dysphonia & $4(9.8)$ & $6(15.0)$ & 0.704 & $0(0.0)$ & $0(0.0)$ & - \\
\hline Edema & $3(7.3)$ & $4(10.0)$ & 0.973 & $0(0.0)$ & $0(0.0)$ & - \\
\hline Elevated uric acid & $3(7.3)$ & $1(2.5)$ & 0.626 & $0(0.0)$ & $0(0.0)$ & - \\
\hline Insomnia & $2(4.9)$ & $2(5.0)$ & 1.000 & $0(0.0)$ & $0(0.0)$ & - \\
\hline Infusion reaction & $2(4.9)$ & - & - & $0(0.0)$ & - & - \\
\hline Hyperglycemia & $1(2.4)$ & $0(0.0)$ & 1.000 & $0(0.0)$ & $0(0.0)$ & - \\
\hline Pneumonitis & $1(2.4)$ & $0(0.0)$ & 1.000 & $1(2.4)$ & $0(0.0)$ & 1.000 \\
\hline
\end{tabular}

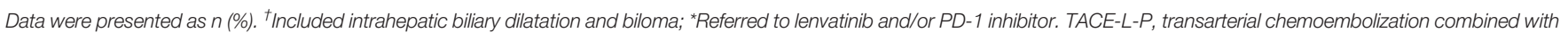

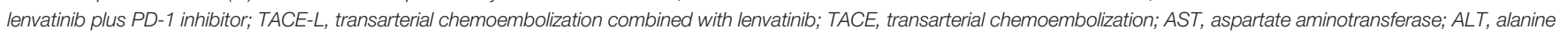
aminotransferase; ALP, alkaline phosphatase; TBi, total bilirubin; GGT, $\gamma$-glutamyl transpeptidase.

Treatment-related AEs led to treatment interruption, dose reduction and treatment discontinuation of lenvatinib in 22 (53.7\%), $21(51.2 \%)$ and $3(7.3 \%)$ patients, respectively, in the TACE-L-P group, and in 20 (50.0\%), 20 (50.0\%) and 3 (7.5\%) patients, respectively, in the TACE-L group. Treatment-related AEs led to treatment interruption and discontinuation of PD-1 inhibitor in $10(24.4 \%)$ and $6(14.6 \%)$ patients in the TACE-L-P group, respectively. Discontinuation of both lenvatinib and PD-1 inhibitor because of AEs occurred in only 2 patients (4.9\%).

\section{DISCUSSION}

Our study showed that TACE-L-P conferred a significant survival benefit when compared with TACE-L in patients with advanced HCC. This finding was associated with an increase in median OS from 12.1 months to 16.9 months, which might be attributed to the higher ORR and DCR and the longer PFS achieved in patients receiving TACE-L-P rather than TACE-L. Multivariate analyses also revealed that combining PD-1 inhibitor on the basis of TACE-L was an independent predictor for prolonged OS and PFS. These results indicated that the triple combination treatment of TACE-L-P might be a superior treatment option in advanced HCC patients. The reasons might be as follows: 1) TACE lead to an extensive local necrosis of the tumor and may subsequently elicit anticancer immune responses that may be further boosted with PD-1 inhibitors $(10,21)$. 2) Lenvatinib is a multikinase inhibitor with antiproliferative and antiangiogenitic activities (22), which may counteract the hypoxia-induced angiogenesis after TACE 
$(9,10)$ and can regulate the tumor immune microenvironment and enhance immune response of PD-1 inhibitor in HCC (22, 23). Therefore, the combination of TACE, lenvatinib and PD-1 inhibitor may bring about a synergistic antitumor activity, contributing to improved clinical outcomes in advanced HCC patients.

Previous studies $(29,30)$ have assessed the combination of TACE, lenvatinib and PD-1 inhibitor for patients with unresectable HCC and reported a PFS of 11.4-13.3 months and an OS of 23.6-24.0 months, which seemed much longer than those for the patients treated with TACE-L-P in our study. However, it was worth noting that these studies enrolled a large proportion (25.0\%-54.5\%) of patients with HCC at BCLC stage B, who were expected to achieve better outcomes than those with BCLC stage C HCC in the present study. Additionally, the heavier tumor burden the patients borne in our study (largest tumor size of $12.3 \pm 4.8 \mathrm{~cm}$ and a considerable proportion of patients with $>3$ intrahepatic tumors, bilobar tumor distribution, main portal vein invasion or extrahepatic metastasis) might also lead to a limited survival benefit of treatment. But then again, compared with TACE-L, TACE-L-P did provide a significant improvement in survival for the HCC patients with advanced disease.

In our study, the presence of main portal vein invasion or extrahepatic metastasis was identified as the independent risk factor for survival. These results were consistent with previous studies (31-34) and further confirmed that main portal vein invasion or extrahepatic spread had a profound adverse effect on prognosis in HCC patients. More notably, in subgroup analyses, a prolonged OS was observed with the treatment of TACE-L-P not in patients with main portal vein invasion but in those without main portal vein invasion, which implied that TACE-L$\mathrm{P}$ might be better employed for HCC patients before the main portal trunk was involved so that an improved survival could be achieved. Furthermore, subgroup analyses also showed that TACE-L-P provided a better OS than TACE-L in the patients with extrahepatic metastasis or tumor number $>3$ but not in those with no extrahepatic metastasis or tumor number $\leq 3$. The reasons might be that TACE exerted its antitumor property mainly by controlling intrahepatic lesions rather than extrahepatic metastases (9) and its effect on multiple tumors was also limited (35). Thus, a treatment strategy combining TACE with a more potent systemic therapy was urgently needed for patients with extrahepatic metastasis or multiple tumors. Our results revealed the necessity of the additional treatment with PD-1 inhibitor to TACE-L for such patients.

In our study, all the AEs with the combination of TACE and lenvatinib with/without PD-1 inhibitor were manageable and consistent with previously reported data on each individual treatment $(7,10,12,36)$. There were no new or unexpected AEs observed. Additionally, the incidence and severity of AEs in TACE-L-P group were comparable to those in TACE-L group. These results suggested that both the treatments of TACE-L-P and TACE-L were tolerable and combining PD-1 inhibitor with TACE-L did not significantly increase the risk of AEs compared with TACE-L, which revealed an acceptable safety profile of TACE-L-P.
In the present study, three different PD-1 inhibitors were used for the treatment of patients in TACE-L-P group. Although our results suggested that the tumor responses, survival and incidence of AEs in the patients treated with sintilimab were similar to those in the patients treated with tislelizumab/ camrelizumab, the inconformity of treatment with these PD-1 inhibitors and its potential effects on treatment outcomes remained to be concerned. Additionally, our study had some other limitations. First, this study was a retrospective study, and the treatment option was individually determined based on the preference of the attending physician and the patient, which inevitably resulted in selection bias. Second, the sample size of this study was limited. The results of subgroup analyses should be cautiously interpreted. Consequently, validation of our findings by further randomized trials is necessary.

In conclusion, our study showed safety and promising outcomes with the treatment of TACE-L-P in patients with advanced HCC. These patients could benefit from TACE-L-P and had markedly better treatment responses and improved survival in comparison with TACE-L. These findings need to be confirmed in large sample, prospective randomized controlled trials.

\section{DATA AVAILABILITY STATEMENT}

The raw data supporting the conclusions of this article will be made available by the authors, without undue reservation.

\section{ETHICS STATEMENT}

The studies involving human participants were reviewed and approved by the Clinical Research and Application Ethics Committee of the Second Affiliated Hospital of Guangzhou Medical University. The patients/participants provided their written informed consent to participate in this study.

\section{AUTHOR CONTRIBUTIONS}

$\mathrm{MC}, \mathrm{WH}$, and $\mathrm{JH}$ conceived and designed the study with supervision from KZ. MC, WH, YG, JWZ, and $\mathrm{KZ}$ provided the provision of study materials or patients. WS, LCL, LTL, BC, YC, and $\mathrm{JZ}$ collected and assembled the data. $\mathrm{MC}, \mathrm{JH}$, and $\mathrm{WS}$ performed or supervised analyses. $\mathrm{MC}, \mathrm{WH}$, and $\mathrm{KZ}$ interpreted the results. $\mathrm{MC}, \mathrm{JH}$, and $\mathrm{KZ}$ wrote the manuscript. All authors reviewed the manuscript and approved the final version.

\section{FUNDING}

This work was supported by the National Natural Science Foundation of China (81873920, 82001930, 82001929), the Science and Technology Project of Guangzhou, China 
(202002030135, 202102010082), the High-Level University Clinical Research Promotion Program of Guangzhou Medical University (B185004019), and the Doctoral Scientific Research Fund of the Second Affiliated Hospital of Guangzhou Medical University (010G271073).

\section{REFERENCES}

1. Sung H, Ferlay J, Siegel RL, Laversanne M, Soerjomataram I, Jemal A, et al. Global Cancer Statistics 2020: GLOBOCAN Estimates of Incidence and Mortality Worldwide for 36 Cancers in 185 Countries. CA Cancer J Clin (2021) 71(3):209-49. doi: 10.3322/caac.21660

2. Zhou J, Sun H, Wang Z, Cong W, Wang J, Zeng M, et al. Guidelines for the Diagnosis and Treatment of Hepatocellular Carcinoma (2019 Edition). Liver Cancer (2020) 9(6):682-720. doi: 10.1159/000509424

3. Benson AB, D'Angelica MI, Abbott DE, Anaya DA, Anders R, Are C, et al. Hepatobiliary Cancers, Version 2.2021, NCCN Clinical Practice Guidelines in Oncology. J Natl Compr Canc Netw (2021) 19(5):541-65. doi: 10.6004/jnccn. 2021.0022

4. European Association for the Study of the Liver. EASL. Clinical Practice Guidelines: Management of Hepatocellular Carcinoma. J Hepatol (2018) 69 (1):182-236. doi: 10.1016/j.jhep.2018.03.019

5. Llovet JM, Ricci S, Mazzaferro V, Hilgard P, Gane E, Blanc JF, et al. Sorafenib in Advanced Hepatocellular Carcinoma. N Engl J Med (2008) 359(4):378-90. doi: 10.1056/NEJMoa0708857

6. Cheng AL, Kang YK, Chen Z, Tsao CJ, Qin S, Kim JS, et al. Efficacy and Safety of Sorafenib in Patients in the Asia-Pacific Region With Advanced Hepatocellular Carcinoma: A Phase III Randomised, Double-Blind, Placebo-Controlled Trial. Lancet Oncol (2009) 10(1):25-34. doi: 10.1016/ S1470-2045(08)70285-7

7. Kudo M, Finn RS, Qin S, Han KH, Ikeda K, Piscaglia F, et al. Lenvatinib Versus Sorafenib in First-Line Treatment of Patients With Unresectable Hepatocellular Carcinoma: A Randomised Phase 3 non-Inferiority Trial. Lancet (2018) 391(10126):1163-73. doi: 10.1016/S0140-6736(18)30207-1

8. Cai M, Li B, Lin L, Huang J, An Y, Huang W, et al. A Reduction and pH DualSensitive Nanodrug for Targeted Theranostics in Hepatocellular Carcinoma. Biomater Sci (2020) 8(12):3485-99. doi: 10.1039/d0bm00295j

9. Kishore SA, Bajwa R, Madoff DC. Embolotherapeutic Strategies for Hepatocellular Carcinoma: 2020 Update. Cancers (Basel) (2020) 12(4):791. doi: $10.3390 /$ cancers 12040791

10. Chang Y, Jeong SW, Young JJ, Jae KY. Recent Updates of Transarterial Chemoembolilzation in Hepatocellular Carcinoma. Int J Mol Sci (2020) 21 (21):8165. doi: 10.3390/ijms21218165

11. Choi GH, Shim JH, Kim MJ, Ryu MH, Ryoo BY, Kang YK, et al. Sorafenib Alone Versus Sorafenib Combined With Transarterial Chemoembolization for Advanced-Stage Hepatocellular Carcinoma: Results of Propensity Score Analyses. Radiology (2013) 269(2):603-11. doi: 10.1148/ radiol.13130150

12. Zhu K, Chen J, Lai L, Meng X, Zhou B, Huang W, et al. Hepatocellular Carcinoma With Portal Vein Tumor Thrombus: Treatment With Transarterial Chemoembolization Combined With Sorafenib-A Retrospective Controlled Study. Radiology (2014) 272(1):284-93. doi: 10.1148/radiol.14131946

13. Fu Z, Li X, Zhong J, Chen X, Cao K, Ding N, et al. Lenvatinib in Combination With Transarterial Chemoembolization for Treatment of Unresectable Hepatocellular Carcinoma (uHCC): A Retrospective Controlled Study. Hepatol Int (2021) 15(3):663-75. doi: 10.1007/s12072-021-10184-9

14. Geschwind JF, Kudo M, Marrero JA, Venook AP, Chen XP, Bronowicki JP, et al. TACE Treatment in Patients With Sorafenib-Treated Unresectable Hepatocellular Carcinoma in Clinical Practice: Final Analysis of GIDEON. Radiology (2016) 279(2):630-40. doi: 10.1148/radiol.2015150667

15. Cheng AL, Hsu C, Chan SL, Choo SP, Kudo M. Challenges of Combination Therapy With Immune Checkpoint Inhibitors for Hepatocellular Carcinoma. J Hepatol (2020) 72(2):307-19. doi: 10.1016/j.jhep.2019.09.025

16. Yau T, Park JW, Finn RS, Cheng A, Mathurin P, Edeline J, et al. CheckMate 459: A Randomized, Multi-Center Phase III Study of Nivolumab (NIVO) vs

\section{SUPPLEMENTARY MATERIAL}

The Supplementary Material for this article can be found online at: https://www.frontiersin.org/articles/10.3389/fimmu.2022. 848387/full\#supplementary-material

Sorafenib (SOR) as First-Line (1L) Treatment in Patients (Pts) With Advanced Hepatocellular Carcinoma (aHCC). Ann Oncol (2019) 30(suppl 5):v874-5. doi: 10.1093/annonc/mdz394.029

17. Finn RS, Ryoo BY, Merle P, Kudo M, Bouattour M, Lim HY, et al. Pembrolizumab As Second-Line Therapy in Patients With Advanced Hepatocellular Carcinoma in KEYNOTE-240: A Randomized, DoubleBlind, Phase III Trial. J Clin Oncol (2020) 38(3):193-202. doi: 10.1200/JCO. 19.01307

18. Finn RS, Qin S, Ikeda M, Galle PR, Ducreux M, Kim TY, et al. Atezolizumab Plus Bevacizumab in Unresectable Hepatocellular Carcinoma. N Engl J Med (2020) 382(20):1894-905. doi: 10.1056/NEJMoa1915745

19. Ren Z, Xu J, Bai Y, Xu A, Cang S, Du C, et al. Sintilimab Plus a Bevacizumab Biosimilar (IBI305) Versus Sorafenib in Unresectable Hepatocellular Carcinoma (ORIENT-32): A Randomised, Open-Label, Phase 2-3 Study. Lancet Oncol (2021) 22(7):977-90. doi: 10.1016/S1470-2045(21)00252-7

20. Finn RS, Ikeda M, Zhu AX, Sung MW, Baron AD, Kudo M, et al. Phase Ib Study of Lenvatinib Plus Pembrolizumab in Patients With Unresectable Hepatocellular Carcinoma. J Clin Oncol (2020) 38(26):2960-70. doi: 10.1200/ JCO.20.00808

21. Cheu JW, Wong CC. Mechanistic Rationales Guiding Combination Hepatocellular Carcinoma Therapies Involving Immune Checkpoint Inhibitors. Hepatology (2021) 74(4):2264-76. doi: 10.1002/hep.31840

22. Kimura T, Kato Y, Ozawa Y, Kodama K, Ito J, Ichikawa K, et al. Immunomodulatory Activity of Lenvatinib Contributes to Antitumor Activity in the Hepa1-6 Hepatocellular Carcinoma Model. Cancer Sci (2018) 109(12):3993-4002. doi: 10.1111/cas.13806

23. Yi C, Chen L, Lin Z, Liu L, Shao W, Zhang R, et al. Lenvatinib Targets FGF Receptor 4 to Enhance Antitumor Immune Response of Anti-Programmed Cell Death-1 in HCC. Hepatology (2021) 74(5):2544-60. doi: 10.1002/hep. 31921

24. Zhou J, Sun HC, Wang Z, Cong WM, Wang JH, Zeng MS, et al. Guidelines for Diagnosis and Treatment of Primary Liver Cancer in China (2017 Edition). Liver Cancer (2018) 7(3):235-60. doi: 10.1159/000488035

25. Liu Y, Huang W, He M, Lian H, Guo Y, Huang J, et al. Efficacy and Safety of CalliSpheres((R)) Drug-Eluting Beads Transarterial Chemoembolization in Barcelona Clinic Liver Cancer Stage C Patients. Oncol Res (2019) 27(5):56573. doi: $10.3727 / 096504018 X 15313896322888$

26. Lencioni R, de Baere T, Burrel M, Caridi JG, Lammer J, Malagari K, et al. Transcatheter Treatment of Hepatocellular Carcinoma With Doxorubicin-Loaded DC Bead (DEBDOX): Technical Recommendations. Cardiovasc Intervent Radiol (2012) 35(5):980-5. doi: 10.1007/s00270-0110287-7

27. Lencioni R, de Baere T, Soulen MC, Rilling WS, Geschwind JF. Lipiodol Transarterial Chemoembolization for Hepatocellular Carcinoma: A Systematic Review of Efficacy and Safety Data. Hepatology (2016) 64 (1):106-16. doi: 10.1002/hep.28453

28. Gaba RC, Lewandowski RJ, Hickey R, Baerlocher MO, Cohen EI, Dariushnia SR, et al. Transcatheter Therapy for Hepatic Malignancy: Standardization of Terminology and Reporting Criteria. J Vasc Interv Radiol (2016) 27(4):45773. doi: 10.1016/j.jvir.2015.12.752

29. Liu J, Li Z, Zhang W, Lu H, Sun Z, Wang G, et al. Comprehensive Treatment of Trans-Arterial Chemoembolization Plus Lenvatinib Followed by Camrelizumab for Advanced Hepatocellular Carcinoma Patients. Front Pharmacol (2021) 12:709060. doi: 10.3389/fphar.2021.709060

30. Cao F, Yang Y, Si T, Luo J, Zeng H, Zhang Z, et al. The Efficacy of TACE Combined With Lenvatinib Plus Sintilimab in Unresectable Hepatocellular Carcinoma: A Multicenter Retrospective Study. Front Oncol (2021) 11:783480. doi: 10.3389/fonc.2021.783480

31. Cheng S, Yang J, Shen F, Zhou W, Wang Y, Cong W, et al. Multidisciplinary Management of Hepatocellular Carcinoma With Portal Vein Tumor 
Thrombus - Eastern Hepatobiliary Surgical Hospital Consensus Statement. Oncotarget (2016) 7(26):40816-29. doi: 10.18632/oncotarget.8386

32. Huang JJ, Cai MY, Huang WS, Guo YJ, Zhou JW, Liang LC, et al. Transarterial Chemoembolization Combined With Sorafenib and Iodine-125 Seed Brachytherapy for Hepatocellular Carcinoma With Portal Vein Tumor Thrombus: A Retrospective Controlled Study. Chin Med J (Engl) (2021) 135 (1):113-5. doi: 10.1097/CM9.0000000000001537

33. Natsuizaka M, Omura T, Akaike T, Kuwata Y, Yamazaki K, Sato T, et al. Clinical Features of Hepatocellular Carcinoma With Extrahepatic Metastases. J Gastroenterol Hepatol (2005) 20(11):1781-7. doi: 10.1111/j.1440-1746. 2005.03919.x

34. Labeur TA, Berhane S, Edeline J, Blanc JF, Bettinger D, Meyer T, et al. Improved Survival Prediction and Comparison of Prognostic Models for Patients With Hepatocellular Carcinoma Treated With Sorafenib. Liver Int (2020) 40(1):215-28. doi: 10.1111/liv.14270

35. Miyayama S, Kikuchi Y, Yoshida M, Yamashiro M, Sugimori N, Ikeda R, et al. Outcomes of Conventional Transarterial Chemoembolization for Hepatocellular Carcinoma $\geq 10$ Cm. Hepatol Res (2019) 49(7):787-98. doi: 10.1111/hepr.13335

36. Sangro B, Chan SL, Meyer T, Reig M, El-Khoueiry A, Galle PR. Diagnosis and Management of Toxicities of Immune Checkpoint Inhibitors in
Hepatocellular Carcinoma. J Hepatol (2020) 72(2):320-41. doi: 10.1016/ j.jhep.2019.10.021

Conflict of Interest: The authors declare that the research was conducted in the absence of any commercial or financial relationships that could be construed as a potential conflict of interest.

Publisher's Note: All claims expressed in this article are solely those of the authors and do not necessarily represent those of their affiliated organizations, or those of the publisher, the editors and the reviewers. Any product that may be evaluated in this article, or claim that may be made by its manufacturer, is not guaranteed or endorsed by the publisher.

Copyright (C) 2022 Cai, Huang, Huang, Shi, Guo, Liang, Zhou, Lin, Cao, Chen, Zhou and Zhu. This is an open-access article distributed under the terms of the Creative Commons Attribution License (CC BY). The use, distribution or reproduction in other forums is permitted, provided the original author(s) and the copyright owner(s) are credited and that the original publication in this journal is cited, in accordance with accepted academic practice. No use, distribution or reproduction is permitted which does not comply with these terms. 